\title{
Lattice Boltzmann model for exterior flows with an annealing preconditioning method
}

\author{
Bo Liu ${ }^{1}$ and Arzhang Khalili ${ }^{1,2, *}$ \\ ${ }^{1}$ Max-Planck Institute for Marine Microbiology, 28359 Bremen, Germany \\ ${ }^{2}$ Jacobs University Bremen, 28759 Bremen, Germany
}

(Received 9 February 2009; revised manuscript received 3 April 2009; published 3 June 2009)

\begin{abstract}
In this paper we propose a highly efficient and stable lattice Boltzmann method for solving low Reynolds number exterior flows using a preconditioning technique. The present method is based on replacing the constant preconditioning parameter $(\gamma)$ within uniform grids [Guo et al., Phys. Rev. E 70, 066706 (2004)] by a space- and time-dependent one in a nested mesh-refined domain. To do this, for the transition from a fine to the neighboring coarser grid, $\gamma$ has been divided by a factor $K$, which is large initially and anneals stepwise to a small value after some iterations. With this technique, more than one order of magnitude larger convergence rate can be achieved, and several orders of magnitude larger system size can be treated.
\end{abstract}

DOI: 10.1103/PhysRevE.79.066701

PACS number(s): 47.11.- j

\section{INTRODUCTION}

Low Reynolds number exterior flows play an increasingly important role in applications, such as marine aggregates [1-4] and sedimentation of small particles in the context of climate prediction $[5,6]$. In some of these applications, e.g., in marine aggregates, often very accurate force computations are required to mimic the real sinking process. Because of the high computational cost involved in the numerical simulation of such flows, schemes providing rapid convergence are highly demanded. The accurate and efficient treatment of these types of flows significantly depends on two issues, being the implementation of far-field conditions and no-slip boundary conditions on the obstacle surface.

To tackle the first issue, Latt et al. [7] and Liu and Khalili [8] showed, respectively, the applicability of the far-field boundary conditions [9] coupled with grid refinement techniques in lattice Boltzmann model (LBM). However, there exists a limit to the number of the refinement level due to the numerical instability associated with LBM, which is set to eight for Bhatnagar-Gross-Krook (or BGK) model. With this limited number, of course, an infinite domain cannot be captured accurately. A remedy to this was provided by tedious extrapolations that lead to rather rough results [8].

As far as the second issue is concerned, the accuracy of the no-slip boundary condition depends on the grid resolution, defined as the number of lattice nodes $(A)$ used to discretize the obstacle height. The higher the value of $A$ becomes, the more accurate will be the solution. In order to maintain a flow at a constant Reynolds number, $A$ can be increased by either enhancing the kinematic viscosity or decreasing the far-field velocity. The first method (Latt et al. [7]) increased the relaxation time, $\tau$, and was found inappropriate because of the strong viscosity-dependent location of the boundary in BGK model, which leads to large errors in under-relaxed situations $(\tau \gg 1)[8,10]$. The second method [8] reduced the Mach number, leading to very slow convergence rates. The reason for this has been known as the consequence of a large disparity between the acoustic wave and

\footnotetext{
*Corresponding author; akhalili@mpi-bremen.de
}

the fluid speed [11]. As a response to the low convergence rate, several acceleration techniques were suggested, including the addition of a false forcing term [12], the implicit discretization of the Boltzmann equation [13], direct solution of linear or nonlinear algebraic systems [14], Mach number annealing method [15], viscosity annealing [16], multigrid method $[17,18]$, and the preconditioned technique $[19,20]$. Among the methods mentioned, only in the preconditioned techniques the simplicity of the standard LBM is maintained. However, both presently available preconditioned methods depart from uniform grids, which are not optimal for external flows.

To overcome both shortcomings, here we propose an efficient method for computation of exterior flows based on an adjustable preconditioned technique for LBM, in which the grid refinement technique is combined with the second-order accurate treatment of the far-field boundary condition. The distinctive feature of the model is that the preconditioned parameter is no longer constant, and varies not only with respect to grid refinement level but also with respect to time.

In contrast to our previous paper [8] where BGK model was used, here we employed the so-called regularized lattice Boltzmann (RLB) proposed by Latt and Chopard [21]. The reason for this choice is as follows. In the D2Q9 lattice, there exists a nine-dimensional moment space, from which six $(\rho$, $\mathbf{u}$, and $\mathbf{I})$ are physically relevant, whereas the other three are not, and termed as "ghost modes" [22]. In BGK, all nine modes are relaxed at the same rate $(\tau)$. However, as shown by Latt and Chopard [21], in RLB it is possible to filter out the ghost modes from the collision operator, and relax them all at the rate "unity." Due to this difference, RLB models gain improved accuracy and numerical stability [21]. The accuracy improvement of RLB has been demonstrated for the Kovasznay flows [23] as well as Womersley flows [24]. A significant improvement of stability by the RLB method was shown for a two-dimensional (2D) cavity flow by Latt and Chopard [23]. Some theoretical background for the duality between hydrodynamic and ghost modes has been recently discussed by Adhikari and Succi [25]. Based on these facts, we preferred the RLB. However, to quantify the differences for the present flow, both models were employed.

The reminder of the manuscript is organized as follows: Section II briefly describes the regularized and precondi- 
tioned lattice Boltzmann model. The numerical implementation including the refinement technique and boundarycondition treatment are brought in Sec. III. Following this, in Sec. IV first, the validation of the model is made, followed by a comparison between BGK and RLB. Second, the criteria for the choice of the preconditioning parameter with respect to space and time are discussed. Here, also a comparison is made between different treatments of the no-slip boundary condition at the obstacle surfaces. Finally some concluding remarks follow in Sec. V.

\section{LATTICE BOLTZMANN MODEL}

\section{A. Regularized lattice Boltzmann model (RLM)}

The lattice Boltzmann dynamics is expressed [21] as

$$
f_{i}\left(\mathbf{x}+\mathbf{e}_{i} \delta_{t}, t+\delta_{t}\right)-f_{i}(\mathbf{x}, t)=-\Omega_{i}(f(\mathbf{x}, t)),
$$

where $f_{i}(x, t)$ is the single-particle distribution function at position $\mathbf{x}$ and time $t$ along the direction represented by the subscript $i$. Furthermore, $\delta t$ is the time increment and $\Omega_{i}$ denotes the collision operator. For the sake of simplicity, we will restrict ourselves to nine-velocities model in two dimensions (D2Q9 model). The discrete velocities are given by $\mathbf{e}_{0}=0$ and $\mathbf{e}_{i}=\lambda_{i}\left(\cos \theta_{i}, \sin \theta_{i}\right) c$, with $\lambda_{i}=1$ and $\theta_{i}=(i$ $-1) \pi / 2$ for $i=1-4$, and $\lambda_{i}=\sqrt{2}$ and $\theta_{i}=(i-5) \pi / 2+\pi / 4$ for $i=5-8$.

To reduce round-off errors, we use the density fluctuation $\delta \rho$ and assume the mean density $\rho_{0}=1$. The total density is therefore $\rho=\rho_{0}+\delta \rho$. In addition, we use flow moment $\mathbf{j}$ $=\rho_{0} \mathbf{u}$ to reduce effects of compressibility $[22,26,27]$. The hydrodynamic variables mass density $(\rho)$, momentum $(\mathbf{j})$ and flux tensor (II) are computed by

$$
\begin{gathered}
\delta \rho=\sum_{i} f_{i}, \\
\mathbf{j}=\rho_{0} \mathbf{u}=\sum_{i} \mathbf{e}_{i} f_{i}, \\
\boldsymbol{\Pi}=\sum_{i} \mathbf{e}_{i} \mathbf{e}_{i} f_{i},
\end{gathered}
$$

The BGK variant of the lattice Boltzmann dynamics expresses the collision as a relaxation toward a local equilibrium, $\Omega_{i}=-\frac{1}{\tau}\left(f_{i}-f_{i}^{e q}\right)$, where $\tau$ is the nondimensional relaxation time directly related to the kinematic fluid viscosity $\nu$ $=c_{s}^{2}\left(\tau-\frac{1}{2}\right) \delta_{t}$ and $f_{i}^{(e q)}$ is the equilibrium distribution function $[22,26,27]$

$$
f_{i}^{(e q)}=\omega_{i}\left[\delta \rho+\rho_{0}\left(\frac{\mathbf{e}_{i} \cdot \mathbf{u}}{c_{s}^{2}}+\frac{\mathbf{u u}:\left(\mathbf{e}_{i} \mathbf{e}_{i}-c_{s}^{2} \mathbf{I}\right)}{2 c_{s}^{2}}\right)\right],
$$

in which $\omega_{i}$ is the weight, $c_{s}$ is the speed of sound (set as $\left.c_{s}^{2}=1 / 3\right)$, and $\mathbf{I}$ is the unit tensor. The weights are given by $\omega_{0}=4 / 9$ and $\omega_{i}=1 / 9$ for $i=1-4$, and $\omega_{i}=1 / 36$ for $i=5-8$.

The key to the regularized procedure is to use an approximation of the nonequilibrium part of the distribution functions $f_{i}^{n e q}$ and momentum flux tensor $\boldsymbol{\Pi}^{\text {neq }}$ as

$$
f_{i}^{n e q}=f_{i}-f_{i}^{e q} \approx f_{i}^{1}=-\frac{\omega_{i} \tau}{c_{s}^{2}} \mathbf{Q}_{i}: \mathbf{S}
$$

$$
\boldsymbol{\Pi}^{n e q}=\boldsymbol{\Pi}-\sum_{i} \mathbf{e}_{i} \mathbf{e}_{i} f_{i}^{e q} \approx \boldsymbol{\Pi}^{1}=\sum_{i} \mathbf{e}_{i} \mathbf{e}_{i} f_{i}^{1}=-2 \rho_{0} c_{s}^{2} \tau \mathbf{S},
$$

where the symmetric tensors $\mathbf{Q}_{i}$ are defined as $\mathbf{Q}_{i}=\mathbf{e}_{i} \mathbf{e}_{i}-c_{s}^{2} \mathbf{I}$ and strain tensor $\mathbf{S}=\frac{1}{2}\left(\nabla \mathbf{u}+(\nabla \mathbf{u})^{T}\right)$. Combining Eqs. (6) and (7), $f_{i}^{1}$ is obtained from

$$
f_{i}^{1}=\frac{\omega_{i}}{2 c_{s}^{4}} \mathbf{Q}_{i}: \mathbf{\Pi}^{n e q},
$$

and the RLM dynamics can be expressed as

$$
\begin{aligned}
f_{i}\left(\mathbf{x}+\mathbf{e}_{i} \delta_{t}, t+\delta_{t}\right)= & f_{i}^{e q}(\mathbf{x}, t)+\left(1-\frac{1}{\tau}\right) \frac{\omega_{i}}{2 c_{s}^{4}} \mathbf{Q}_{i}: \sum_{j} \mathbf{e}_{i} \mathbf{e}_{i}\left[f_{j}(\mathbf{x}, t)\right. \\
& \left.-f_{j}^{e q}(\mathbf{x}, t)\right],
\end{aligned}
$$

More details on the regularized lattice Boltzmann model and a discussion of its relation to two or multirelaxation-time models [28-30] can be found elsewhere [21,25]

\section{B. Preconditioned lattice Boltzmann model}

In the classic preconditioned LBM [19], the equilibrium distribution is defined by

$$
f_{i}^{(e q)}=\omega_{i}\left[\delta \rho+\rho_{0}\left(\frac{\mathbf{e}_{i} \cdot \mathbf{u}}{c_{s}^{2}}+\frac{\mathbf{u u}:\left(\mathbf{e}_{i} \mathbf{e}_{i}-c_{s}^{2} \mathbf{I}\right)}{2 \gamma c_{s}^{2}}\right)\right],
$$

with $0<\gamma \leq 1$ as the preconditioning parameter. Through the Chapman-Enskog procedure, we can derive the macroscopic equations as

$$
\frac{\partial \rho}{\partial t}+\nabla \cdot\left(\rho_{0} \mathbf{u}\right)=0
$$

$$
\frac{\partial\left(\rho_{0} \mathbf{u}\right)}{\partial t}+\frac{1}{\gamma} \nabla \cdot\left(\rho_{0} \mathbf{u u}\right)=-\frac{1}{\gamma} \nabla p^{*}+\frac{1}{\gamma} \nabla \cdot\left(2 \rho_{0} \nu^{*} \mathbf{S}\right),
$$

where the effective pressure and viscosity can be given [19] by

$$
\begin{gathered}
p^{*}=\gamma c_{s}^{2} \delta \rho, \\
\nu^{*}=\gamma c_{s}^{2}\left(\tau-\frac{1}{2}\right) \delta_{t} .
\end{gathered}
$$

In the presently available preconditioning techniques, uniform grids and a constant preconditioning parameter were used. However, for the treatment of the far-field boundaryconditions nested grids become necessary [8], and constant preconditioning parameter cannot be used over the entire grids any more. The method suggested here is an extension of the previous models in the sense that the preconditioning parameter varies over both the space as well as time. The details are explained in Sec. IV.

\section{Intergrid transformation}

To ensure the continuity of pressure, velocity, and also of their derivatives at the interface between coarse and fine 


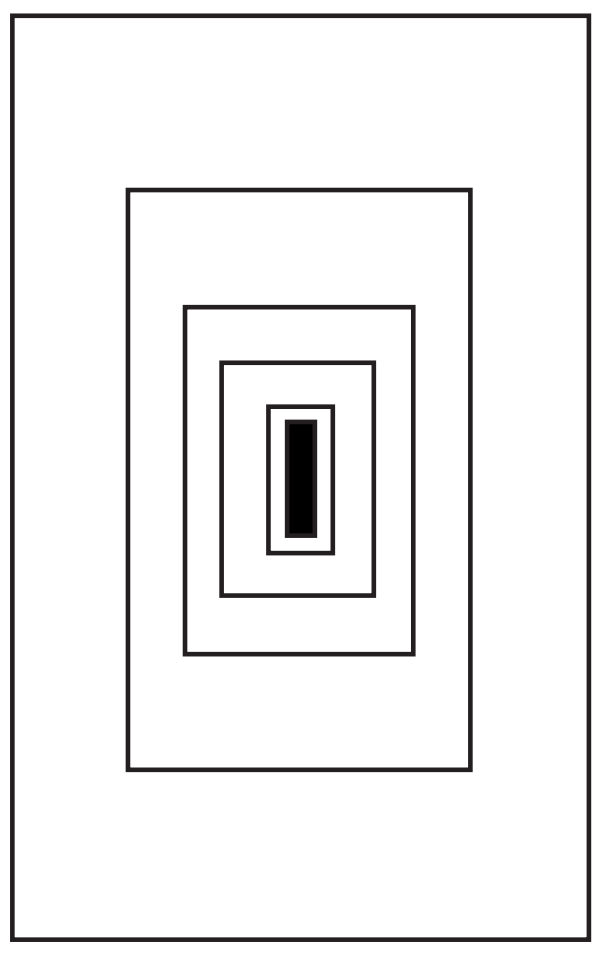

FIG. 1. Structure of the numerical grid close to a rectangular obstacle (black vertical box). The empty boxes from center outward denote the coarsening levels. The flow direction is from left to right.

grids, the missing distributions on one refinement level have to be computed from the adjacent grids. In the usual BGK procedure the distribution functions $\left(f^{e q}\right)$ and $\left(f^{n e q}\right)$ are interpolated [31-34]. Alternatively, the missing distribution functions can also be obtained by interpolation of density, velocity, and flux tensor, combined with appropriate asymptotic relations [35]. However, the use of RLB is more convenient as the interpolation acts on density, velocity, and flux tensor directly by

$$
\begin{gathered}
\gamma_{c} \delta \rho_{c}=\gamma_{f} \delta \rho_{f}, \\
\mathbf{u}_{c}=\mathbf{u}_{f}, \\
\boldsymbol{\Pi}_{c}^{1} /\left(\delta_{t}^{c} \tau_{c}\right)=\boldsymbol{\Pi}_{f}^{1} /\left(\delta_{t}^{f} \tau_{f}\right),
\end{gathered}
$$

with $c$ and $f$ denoting the coarser and finer grids, respectively. In addition, a cubic spline interpolation in space and a local time-stepping scheme have been used as described elsewhere [8].

\section{NUMERICAL IMPLEMENTATION}

The geometry studied here is a rectangular solid block shown in Fig. 1. The details of the parameters influencing the flow are identical to those in previous literature $[7,8]$, and are given by height to width ratio (5/1) and Reynolds number

$$
\operatorname{Re}=A u_{\infty} / \nu,
$$

set to unity.

A grid refinement technique is applied with a hierarchy of nested grids that have a successively finer resolution as they approach the system center. In the finest grid, the spatial and temporal step sizes $\left(\delta_{x}\right.$ and $\left.\delta_{t}\right)$ have been set to unity, while they are multiplied by two as we move from finer to coarser grids. This choice makes the velocity $\mathbf{u}$ to be the same from one refinement level to another, known as convective scaling $(\delta t \sim \delta x)$. Although the diffusive scaling $\left(\delta t \sim \delta x^{2}\right)$ leads to the second-order accurate time interpolation while the convective scaling leads to a first-order one [35], the accuracy of the time interpolation is not crucial when a stationary flow is considered. More importantly, the velocity $\mathbf{u}$ has to be kept small, reflecting a low Mach number flow in LBM. In a diffusive scaling the velocity $\mathbf{u}$ is doubled with every gridcoarsening step. Due to implementation of the far-field boundary condition, a large number of grid-coarsening levels $\left(N_{g}\right)$ has to be implemented. The implementation of a diffusive scaling would have led to large velocities $\mathbf{u}=2^{N_{g}-1} \mathbf{u}_{0}$ in the coarsest grids ( $\mathbf{u}_{0}$ : velocity in the finest grid level), which would have violated the low Mach number assumption. All simulations were run on rectangular domains of varying refinement levels. In each refinement level, the system sizes were $129 \times 129,257 \times 257,257 \times 513$, and $257 \times 1025$ corresponding to $A=80,160,320,640$, respectively. The relaxation time $\tau$ in the finest grid was set to unity.

As the boundary conditions at the obstacle surface we have compared the implementation of eight popular schemes, namely full-way bounce back (FBBC), half-way bounce back (HBBC), equilibrium method (EM) [8], Inamuro [36], Zou-He [37], Halliday [38], Hollis [39], and Latt's regularized method [40]. At the infinity, the secondorder accurate far-field boundary condition [9] was implemented using nonlocal regularized schemes Latt et al. [40]. The momentum-exchange method was employed to evaluate the force on the obstacle.

\section{RESULTS AND DISCUSSION}

\section{A. Model validation}

In order to demonstrate the correctness and the continuity of the interpolation method [Eqs. (15)-(17)], the contours of pressure, the vertical velocity component $\left(u_{y}\right)$, and strains $S_{x x}$ and $S_{x y}$ have been plotted in Fig. 2. The three dashed boxes around the obstacle (gray rectangle) shown in the figure denote interfaces of different grid refinement levels. As can be depicted in the figure, all contours display perfect continuity properties. The input parameters for this case were $A=640$, a system size of $257 \times 1025$, a total grid-coarsening level of 15. As our main concern was the demonstration of the continuous behavior of $p, u_{y}, S_{x x}$, and $S_{x y}$, the values of the contour lines were not shown.

Using the velocity gradients and the pressure, we have obtained the drag coefficient $d$, which was used to compare the accuracy of our computations versus existing reference values [9]. Compared to the reference value $d=2.5145$ we computed $d=2.51451$, leading to a negligible relative error.

As mentioned in Sec. I, there have been some works demonstrating the superiority of RLB over BGK in terms of accuracy and stability for some flow types. In the context of this study, we employed both models to have a quantitative evaluation of this issue. The results showed that the accuracy 


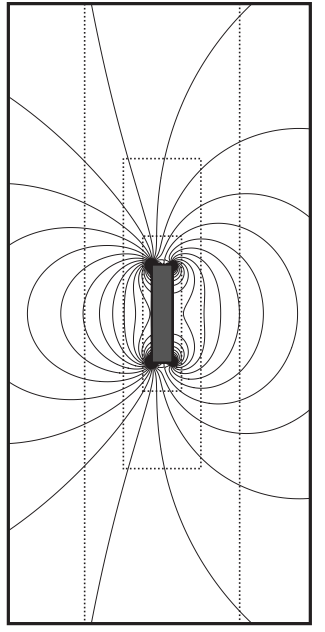

(a)

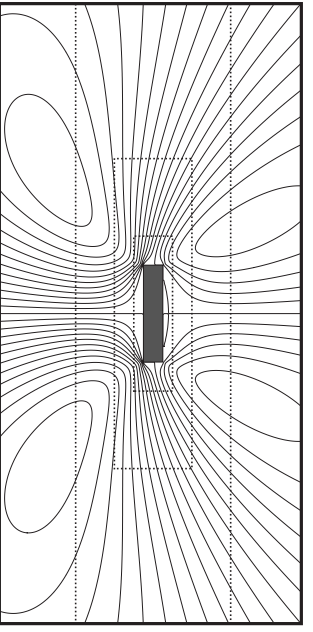

(b)

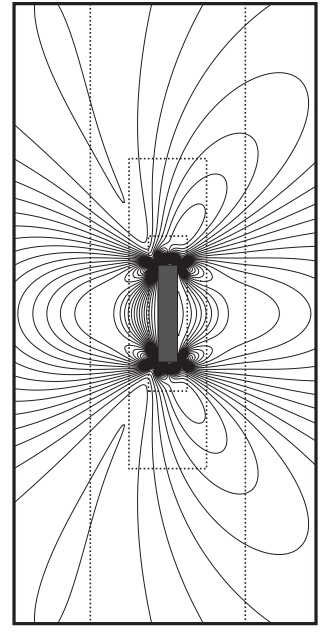

(c)

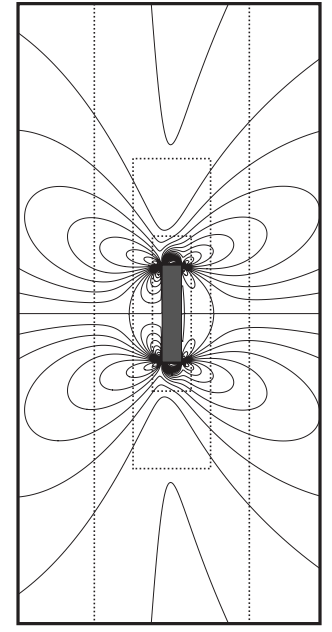

(d)

FIG. 2. From left to right, the contours of $P, y$ velocity $\left(u_{y}\right)$, and strain $\left(S_{x x}\right.$ and $\left.S_{x y}\right)$ near the obstacle (four grids refinement level). The dotted lines represent the interfaces of different grids for $A=640$, a system size of $257 \times 1025$, and a total refinement level of 15 .

gain of RLB was minor compared to that of BGK. However, as far as the stability is concerned, RLB had an increased performance. Consequently, in RLB, one grid refinement level more than in BGK could be implemented.

\section{B. Criterion for the choice of the preconditioning parameter $\gamma$}

The acceleration rate of preconditioned LBM depends on the preconditioning parameter $\gamma$. Here, the selection criterion for $\gamma$ has been adopted from Guo et al. [19], and is given by $\gamma=\left(M / M^{*}\right)^{2}$ with the effective Mach number $M^{*}$ being larger than the standard Mach number $M$. In order to obtain faster convergence rate, a large $M^{*}$ is preferred. However, from linear stability analysis [19], it follows that there exits a critical value $M_{c}^{*}$, above which the preconditioned lattice Boltzmann equation becomes unstable. Therefore, $M^{*}$ can be chosen as the value just below $M_{c}^{*}$, which can be obtained numerically. When uniform grids are chosen, constant $\gamma$ values [slightly larger than $\gamma_{c}=\left(M / M_{c}^{*}\right)^{2}$ ] are preferred in order to accelerate the convergence rate. However, as mentioned above for problems involving a grid refinement procedure, the preconditioning factor $\gamma$ cannot be kept constant for all grid levels. Here, a gradual variation in $\gamma$ from finest (upper $\gamma$ limit) to coarsest grid level (lower $\gamma$ limit), respectively, is necessary. In the finest grid level no preconditioning is required, i.e., $\gamma=1$, which corresponds to the upper limit of $\gamma$. This implies $\left(\tau^{*}=\tau=1\right)$, providing best accuracy for no-slip boundary condition [8]. However, in the coarsest refinement level, the lower $\gamma$ limit was chosen slightly larger than $\gamma_{c}$. The transition from fine to coarse refinement levels is made through dividing $\gamma$ by an annealing factor $K\left(K=\gamma_{i} / \gamma_{i+1}\right.$ with $i$ denoting the coarsening level) until the lower $\gamma$ limit has been reached. In order to compare the results under otherwise similar conditions, only eight refinement levels were taken. This corresponds to the maximum possible refinement level in the absence of preconditioning [8]. Note that in each refinement level, $257 \times 257$ square nodes were used for $A$ $=160$. As demonstrated in Fig. 3, the convergence rate of the preconditioned method is almost one order of magnitude faster than that of the standard method.

Another advantage of preconditioning technique is that an increase in the effective viscosity [19] leads to an improvement of the stability of LBM and, subsequently, to an increase in the maximum number of the refinement levels required when a highly accurate computation is in demand. The vertical dotted line in Fig. 4 corresponds to a grid refinement level of eight without preconditioning in previous studies. As shown in the figure, rather large relative errors in the drag coefficients are made, which range from $9.9 \%$, $1.4 \%$, and $0.69 \%$ corresponding, respectively, to the zeroth, first- and second-order treatment of the far-field boundaries. By contrast, when our preconditioning is used, the relative errors can be reduced to $6.1 \times 10^{-4}, 1.3 \times 10^{-5}$, and 1.3 $\times 10^{-6}$, when 15 refinement levels have been employed [Fig. $4(b)]$. Note that in each refinement level a total node number of $257 \times 1025$ were implemented.

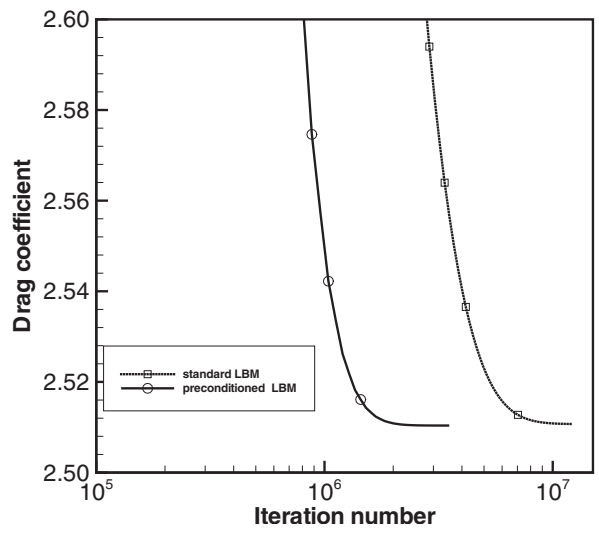

FIG. 3. Convergence histories of the drag coefficients $d$ using standard and preconditioned LBM with $K=2$. The refinement level was fixed to 8 . In each refinement level, $257 \times 257$ square nodes were used for $A=160$; equilibrium method for no-slip boundary condition on the obstacle surface was used. The figure shows clearly that much less iteration numbers are required by preconditioned LBM. 

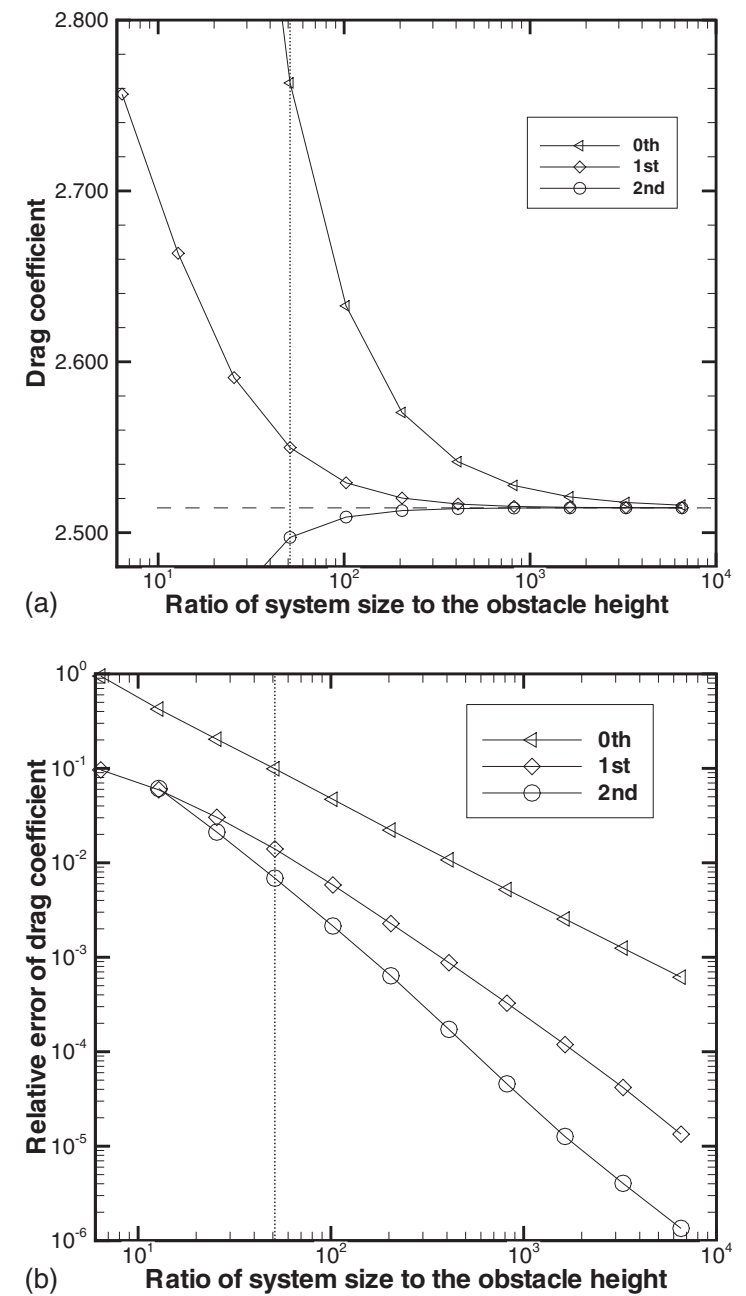

FIG. 4. Drag coefficient $d$ (a) and its relative error (b) as a function of the system size with asymptotic far-field boundary conditions of the zeroth, first, and second order. A total of $257 \times 1025$ nodes in each refinement level with $A=640$ and the equilibrium method for no-slip boundary condition on the obstacle surface were used. The vertical dotted line in the figure denotes eight refinement levels. All symbols right to this line correspond to higher levels which minimize the relative errors. The horizontal dashed line in panel (a) denotes the reference solution $d=2.5145$ of Bönisch et al. [9].

\section{Annealing factor $K$}

In addition to the preconditioning with respect to space (mentioned above), here a preconditioning with respect to time is introduced. As can be seen from Fig. 5, constant $K$ 's either lead to more accurate results at the cost of more iteration numbers $(K=2)$ or less iteration number sacrificing the accuracy $(K=8)$. In the case of $K=2$, namely, a constant $\tau$ $=1$ for all subsequent coarsening levels can be reached [a consequence of $\tau_{f}=\tau_{c}=1$ from Eq. (14)], and has been known to result in best accuracies in BGK-type methods. Furthermore, in the case of $K=8$ less iteration numbers are required due to larger effective Mach number for coarser grid levels (resulting from lower preconditioning parameter, $\gamma$ ). Note that a further decrease in $K$ from 2 to $K=1.5$ does not lead to a significant accuracy change, and requires rather

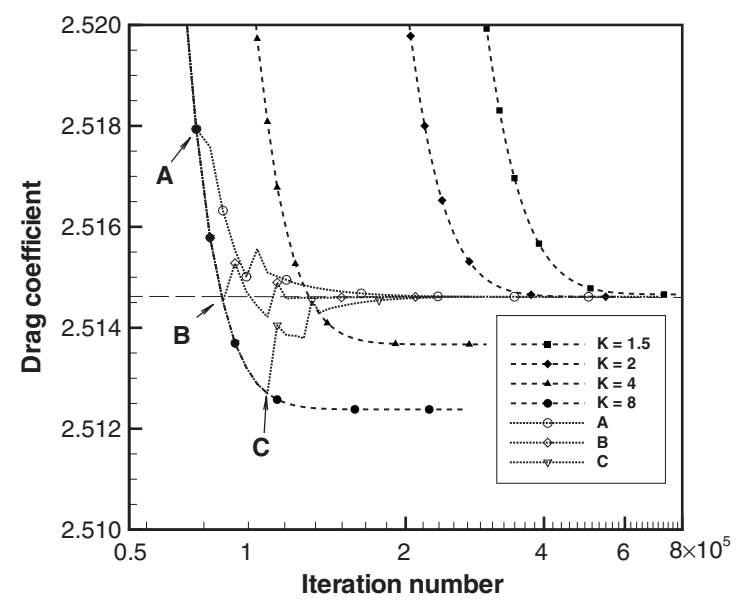

FIG. 5. Convergence histories of the drag coefficients $d$ using preconditioned LBM with different $K$. All short-dashed curves were produced with constant $K$ 's, whereas those denoted by A, B, and C were generated using annealing $K$ 's. The refinement level was fixed to 13 . A total of $257 \times 513$ nodes in each refinement level, with $A$ $=320$ and the equilibrium method for no-slip boundary condition on the obstacle surface were used. The horizontal dashed line in the figure denotes the reference solution $d=2.5145$.

more computational time. In order to maintain both the efficiency and accuracy simultaneously, a variable $K$ is implemented here. By taking a large $K$ (i.e., $K=8$ ) initially and annealing it stepwise, e.g., to 4 and then to 2 after some iterations, best performance can be achieved (Fig. 5) as demonstrated by the dotted line.

Since the reference solution is usually not known a priori, a $K$ annealing can be applied when a certain error bound, $\epsilon$, has been reached within two subsequent time steps. It is obvious that extremely small and large $\epsilon$ 's lead to poor convergence rates. In the lack of any theoretical base for the choice of the annealing criterion, $\epsilon$ can be chosen by numerical approximation. Here, we have set a residual bound of 1 $\times 10^{-5}$. In Fig. 5 three examples are given with $\epsilon=4 \times 10^{-5}$, $2 \times 10^{-5}$, and $1 \times 10^{-5}$, respectively, corresponding to the points $\mathrm{A}, \mathrm{B}$, and $\mathrm{C}$. As can be seen from the figure, the performance of the annealing procedure is superior to those with constant $K$ 's. Rigorous numerical tests showed that the results did not depend significantly on the choice of the position (A, B, or C), from which the annealing process was introduced.

\section{TREATMENT OF NO-SLIP BOUNDARY CONDITIONS}

For the treatment of the no-slip boundary condition at the surface of a solid obstacle two different methods can be employed. The first one is the so-called bounce-back rule and its modified version [41-44]. However, all these schemes reduce to HBBC once the solid wall is located in the middle of two subsequent grid points, which is the case in the present study. Therefore, we need to consider the HBBC FBBC rule here. In the second method, one sets equilibrium distribution functions (EM) and its modifications suggested by Inamuro [36], Zou-He [37], Halliday [38], Hollis [39], and Latt [40]. 

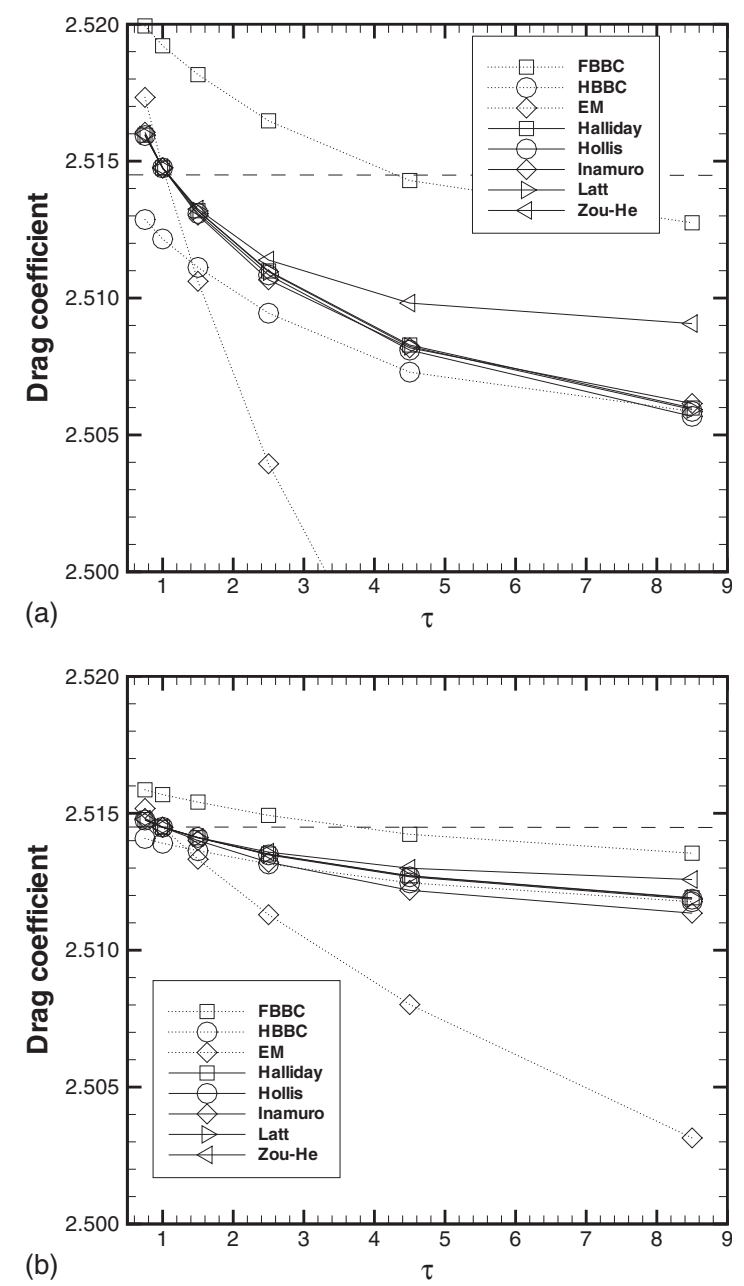

FIG. 6. Drag coefficient $d$ as a function of relaxation time $\tau$ with $A=160$ (a) and $A=640$ (b). The FBBC, HBBC, EM, Halliday, Hollis, Inamuro, Latt, and Zou-He schemes were implemented for the treatment of the no-slip boundary condition at the obstacle surface. The horizontal dashed line represents the reference solution with $d=2.5145$.

A summary of the comparison of these different possibilities can be found elsewhere [8].

Here we employ all these schemes to compare their impact on the accuracy of drag calculations. The implementation of the no-slip boundary conditions on the obstacle surface depends strongly on the relaxation time $\tau$. It was found that for $\tau=1$ the methods in the second category reduce to EM, which has a better accuracy when compared to HBBC and FBBC. For $\tau>1$, Zou-He method showed a better accuracy among all others. All these results, however, depend on $A$ (equivalent to the domain extend), which was rather insufficient and limited to a maximum of $A=160$ in previous studies. A further increase in $A$ was found to be difficult due to the known effects mentioned in the introduction.

Using the preconditioning technique explained above, it is possible to increase the extend of $A$ beyond this limit and to enhance the accuracy of the computations. This effect has been shown in Fig. 6 for two different grid resolutions of the obstacle height $(A=160$ and 640). As can be clearly seen, an increase of $A$ results in drag coefficients being much closer

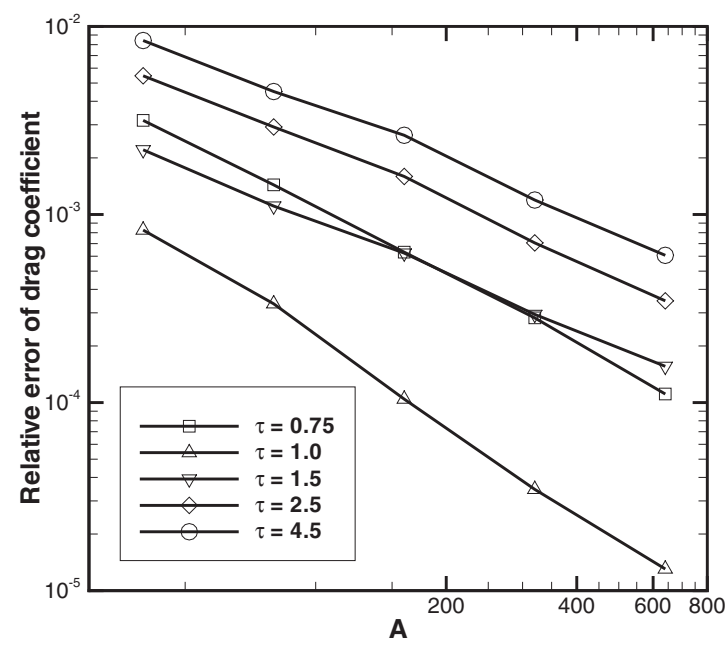

FIG. 7. Relative error of the drag as a function of $A$. Zou-He scheme was implemented for the treatment of the no-slip boundary condition at the obstacle surface. As demonstrated, best results were obtained with $\tau=1$.

to the reference value when $A$ is enhanced. Although, for all methods (except FBBC) the optimal relaxation time is approximately unity, the improvement obtained by increasing $A$ holds for all values of $\tau$.

It is also visible from the results that all methods (except $\mathrm{FBBC}$ and $\mathrm{HBBC}$ ) reduce to the equilibrium method (EM) when $\tau=1$. As can be confirmed by the results, the equilibrium-based methods do not differ for $\tau<1$, whereas for $\tau>1$ Zou-He method performs best. In this case a quantitative evidence can be found when plotting the relative error of the drag coefficient versus $A$ for different values of relaxation time.

Figure 7 indicates clearly that the relative error of the drag coefficient increases as $\tau$ moves away from unity. Also, the relative error of the drag coefficient, e.g., for $\tau=1$, reduces approximately one order of magnitude when $A$ is increased from 160 to 640 , showing the $A$ dependency of the accuracy of the solutions obtained. Finally, the figure also shows that changing $\tau$ from 4.5 to 1.0 decreases the relative error almost two orders of magnitude.

\section{CONCLUSIONS}

In the present study, an improved preconditioned lattice Boltzmann method has been introduced. The suggestion is based on a space- and time-dependent preconditioning parameter $(\gamma)$. Unlike previous studies, here $\gamma$ has been divided by a factor $K$ for the transition from each fine grid to the neighboring coarser one. The investigation showed that large values of $K$ (e.g., 8) result in a rapid convergence whereas small values of $K$ (e.g., 2) lead to an increased accuracy. Therefore, to keep both advantages simultaneously, $K$ has been annealed stepwise from an initially large value to a small one at the final stage.

The comparison between the nonpreconditioned and preconditioned LBM has shown that the latter one provides a 
significant increase of efficiency and stability. Furthermore, the annealing procedure has proved to be superior to a nonannealing method. The improved stability enables us to handle much larger system sizes than possible in nonpreconditioned methods, which were limited to eight coarsening levels. The accurate drag coefficient obtained with this method allows us to evaluate the performance of different techniques for treating the no-slip boundary conditions at obstacle surfaces. The results showed that Zou-He's method performed best among eight different methods compared here.

Finally, we should note the following: although we used a simple benchmark example (flow past a solid rectangular obstacle) here, the method can directly be applied to other complex obstacle geometries and extended to multirelaxation-time methods.
[1] T. Kiørboe, H. Plough, and U. H. Thygesen, Mar. Ecol.: Prog. Ser. 211, 1 (2001).

[2] T. Kiørboe and U. H. Thygesen, Mar. Ecol.: Prog. Ser. 211, 15 (2001).

[3] T. Kiørboe and G. A. Jackson, Limnol. Oceanogr. 46, 1309 (2001).

[4] S. Bhattacharyya, S. Dhinakaran, and A. Khalili, Chem. Eng. Sci. 61, 4451 (2006).

[5] M. B. Baker, Science 276, 1072 (1997).

[6] H. R. Pruppacher and J. D. Klett, Microphysics of Clouds and Precipatitation (Kluwer, Dordrecht, 2000).

[7] J. Latt, Y. Grillet, B. Chopard, and P. Wittwer, Math. Comput. Simul. 72, 169 (2006).

[8] B. Liu and A. Khalili, Phys. Rev. E 78, 056701 (2008).

[9] S. Bönisch, V. Heuveline, and P. Wittwer, J. Math. Fluid Mech. 10, 45 (2008).

[10] C. Pan, L.-S. Luo, and C. T. Miller, Comput. Fluids 35, 898 (2006).

[11] E. Turkel, Annu. Rev. Fluid Mech. 31, 385 (1999).

[12] D. Kandhai, A. Koponen, A. Hoekstra, M. Kataja, J. Timonen, and P. M. A. Sloot, J. Comput. Phys. 150, 482 (1999).

[13] T. Lee and C.-L. Lin, J. Comput. Phys. 185, 445 (2003).

[14] M. Bernaschi, S. Succi, and H. Chen, J. Sci. Comput. 16, 135 (2001).

[15] A. Artoli, A. Hoekstra, and P. Sloot, Int. J. Mod. Phys. C 14, 835 (2003).

[16] M. Bernaschi and S. Succi, Int. J. Mod. Phys. B 17, 1 (2003).

[17] J. Tölke, M. Krafczyk, and E. Rank, J. Stat. Phys. 107, 573 (2002).

[18] D. J. Mavriplis, Comput. Fluids 35, 793 (2006).

[19] Z. Guo, T. S. Zhao, and Y. Shi, Phys. Rev. E 70, 066706 (2004).

[20] S. Izquierdo and N. Fueyo, Prog. Comput. Fluid Dyn. 8, 189 (2008).

[21] J. Latt and B. Chopard, Math. Comput. Simul. 72, 165 (2006).

[22] P. J. Dellar, J. Comput. Phys. 190, 351 (2003).
[23] J. Latt and B. Chopard, e-print arXiv:physics/0506157.

[24] L. Axner, A. G. Hoekstra, and P. M. A. Sloot, Int. J. Mod. Phys. C 18, 661 (2007).

[25] R. Adhikari and S. Succi, Phys. Rev. E 78, 066701 (2008).

[26] Q. Zou, S. Hou, and G. D. Doolen, J. Stat. Phys. 81, 319 (1995).

[27] X. He and L.-S. Luo, J. Stat. Phys. 88, 927 (1997).

[28] I. Ginzburg, F. Verhaeghe, and D. d'Humières, Comm. Comp. Phys. 3, 427 (2008).

[29] I. Ginzburg, F. Verhaeghe, and D. d'Humières, Comm. Comp. Phys. 3, 519 (2008).

[30] P. Lallemand and L.-S. Luo, Phys. Rev. E 61, 6546 (2000).

[31] A. Dupuis and B. Chopard, Phys. Rev. E 67, 066707 (2003).

[32] D. Yu, R. Mei, and W. Shyy, Int. J. Numer. Methods Fluids 39, 99 (2002)

[33] O. Filippova and D. Hänel, J. Comput. Phys. 165, 407 (2000).

[34] B. Crouse, E. Rank, M. Krafczyk, and J. Tölke, Int. J. Mod. Phys. B 17, 109 (2003).

[35] M. Junk, A. Klar, and L.-S. Luo, J. Comput. Phys. 210, 676 (2005).

[36] T. Inamuro, M. Yoshino, and F. Ogino, Phys. Fluids 7, 2928 (1995).

[37] Q. Zou and X. He, Phys. Fluids 9, 1591 (1997).

[38] I. Halliday, L. Hammond, and C. Care, J. Phys. A 35, L157 (2002).

[39] A. Hollis, I. Halliday, and C. Care, J. Phys. A 39, 10589 (2006).

[40] J. Latt, B. Chopard, O. Malaspinas, M. Deville, and A. Michler, Phys. Rev. E 77, 056703 (2008).

[41] M. Bouzidi, M. Firdaouss, and P. Lallemand, Phys. Fluids 13, 3452 (2001).

[42] O. Filippova and D. Hänel, J. Comput. Phys. 147, 219 (1998).

[43] R. Mei, L.-S. Luo, and W. Shyy, J. Comput. Phys. 155, 307 (1999).

[44] M. Junk and Z. Yang, J. Stat. Phys. 121, 3 (2005). 